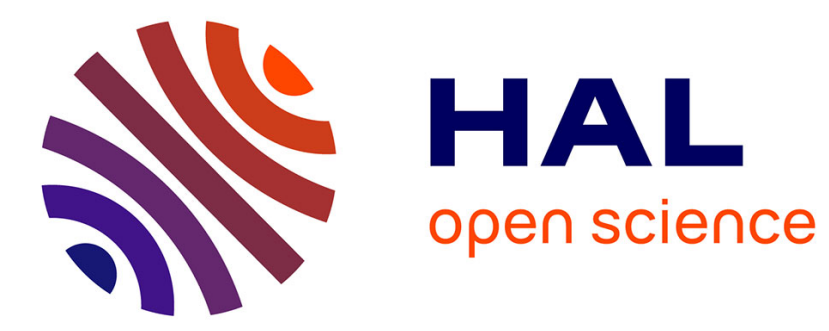

\title{
Subdifferentials of the Marginal Functions in Parametric Convex Optimization via Intersection Formulas
}

\author{
Duong Thi Viet An, Abderrahim Jourani
}

\section{To cite this version:}

Duong Thi Viet An, Abderrahim Jourani. Subdifferentials of the Marginal Functions in Parametric Convex Optimization via Intersection Formulas. Journal of Optimization Theory and Applications, 2022, 192 (1), pp.82-96. 10.1007/s10957-021-01952-6 . hal-03448368

\section{HAL Id: hal-03448368 https://hal.science/hal-03448368}

Submitted on 25 Nov 2021

HAL is a multi-disciplinary open access archive for the deposit and dissemination of scientific research documents, whether they are published or not. The documents may come from teaching and research institutions in France or abroad, or from public or private research centers.
L'archive ouverte pluridisciplinaire HAL, est destinée au dépôt et à la diffusion de documents scientifiques de niveau recherche, publiés ou non, émanant des établissements d'enseignement et de recherche français ou étrangers, des laboratoires publics ou privés. 


\section{Subdifferentials of the Marginal Functions in Parametric Convex Optimization via Intersection Formulas}

D. T. V. $A n^{12}$ and A. Jourani ${ }^{3}$

\footnotetext{
${ }^{1}$ School of Management, Hangzhou Dianzi University, Hangzhou 310018, China. Email: andtv@hdu.edu.cn.

${ }^{2}$ Department of Mathematics and Informatics, Thai Nguyen University of Sciences, Thai Nguyen city, Vietnam. Email: andtv@tnus.edu.vn.

${ }^{3}$ Institut de Mathématiques de Bourgogne, UMR 5584 CNRS Université de Bourgogne Franche-Comté, Dijon Cedex, France. Email: Abderrahim.Jourani@u-bourgogne.fr.
} 


\begin{abstract}
The aim of the present work is to use a metric intersection formula to estimate the subdifferential of the marginal function in the convex setting. This intersection formula includes many interesting situations in parametric convex programming, including the polyhedral one. It is expressed in terms of the objective function and the constrained multivalued mapping which govern the parametric program.
\end{abstract}

Key Words. Parametric convex programming; marginal function; subdifferential; singular subdifferential; distance function

AMS Subject Classifications: 49J53; 49Q12; 90C25; 90C31. 


\section{Introduction}

Intersection formulas play an important role in optimization and variational and nonsmooth analysis. For example it allows to obtain the relationship between normal cone to set intersections and normal cone to each set in the intersection, to get chain rules for subdifferentials, and to estimate the subdifferential of the marginal function (see [16]). To be concrete, we say that two closed sets $C$ and $D$ of a normed space $X$ satisfy the intersection formula at $x_{0} \in C \cap D$ if there exist two real numbers $a>0$ and $r>0$ such that

$$
d(x, C \cap D) \leq a(d(x, C)+d(x, D)) \quad \forall x \in \mathbb{B}\left(x_{0}, r\right),
$$

where $x \mapsto d(x, B)$ denotes the distance function to a set $B \subset X$.

Normal cones to the intersection. Condition (1) ensures the following inclusion

$$
N\left(x_{0} ; C \cap D\right) \subset N\left(x_{0} ; C\right)+N\left(x_{0} ; D\right)
$$

where $N$ denotes either the Fenchel cone in the sense of convex analysis with $C$ and $D$ being closed convex sets or the limiting Fréchet normal cone (see [18]) whenever $X$ is a Asplund space or the nucleus normal cone (see [14]) whenever $X$ is any Banach space. Unfortunately, the intersection formula (1) is not sufficient to guarantee the same chain rule for Clarke's normal cone. However, for the latter one, we obtain

$$
N\left(x_{0} ; C \cap D\right) \subset \mathrm{cl}^{*}\left[N\left(x_{0} ; C\right)+N\left(x_{0} ; D\right)\right]
$$

where $\mathrm{cl}^{*}(A)$ denotes the weak-star closedness of $A \subset X^{*}$ and $X^{*}$ is the dual space of $X$.

Subdifferential calculus. Consider the sets

$$
S_{1}=\left\{(x, \alpha, \beta) \in X \times \mathbb{R} \times \mathbb{R}: f_{1}(x) \leq \alpha\right\}
$$

and

$$
S_{2}=\left\{(x, \alpha, \beta) \in X \times \mathbb{R} \times \mathbb{R}: f_{2}(x) \leq \beta\right\},
$$


for extended real-valued functions $f_{1}$ and $f_{2}$ on $X$. We say that $f_{1}$ and $f_{2}$ satisfy the intersection formula at $x_{0}$ if there exist $r>0$ and $a>0$ such that

$$
d\left((x, \alpha, \beta), S_{1} \cap S_{2}\right) \leq a\left[d\left((x, \alpha, \beta), S_{1}\right)+d\left((x, \alpha, \beta), S_{2}\right)\right]
$$

for all $x \in x_{0}+r \mathbb{B}_{X}, \alpha \in f_{1}\left(x_{0}\right)+r \mathbb{B}_{\mathbb{R}}$ and $\beta \in f_{2}\left(x_{0}\right)+r \mathbb{B}_{\mathbb{R}}$. Condition (2) leads to the following chain rule

$$
\partial\left(f_{1}+f_{2}\right)\left(x_{0}\right) \subset \partial f_{1}\left(x_{0}\right)+\partial f_{2}\left(x_{0}\right)
$$

where $\partial$ denotes either the Fenchel subdifferential with $f_{1}$ and $f_{2}$ being convex functions or the limiting Fréchet subdifferential (see [18]) whenever $X$ is a Asplund space or the nucleus subdifferential (see [14]) whenever $X$ is any Banach space.

The study of the behavior of the marginal (or value) function is known to be very important in variational analysis and optimization theory; see, e.g., $[1,2,3,5,11,12,16,19,20,21]$ and the references therein.

In [16], the author has proposed the method by using intersection formulas to evaluate the $G$-subdifferentials of the marginal functions of parametric programming problems under inclusion constraints in Banach spaces. Recently, by using the Moreau-Rockafellar theorem and appropriate regularity conditions, An and Yen [2], An and Yao [1] have obtained formulas for computing subdifferentials of the marginal functions for convex optimization problems under inclusion constraints on infinite dimensional spaces.

The primary goal of this paper is to use intersection formulas for estimating subdifferentials of the marginal functions of convex parametric programming problems under inclusion constraints in Banach spaces. Thus, on one hand, our results have the origin in the study of [16]. On the other hand, they are the results of deepening that study for the case of convex programming problems.

The contents of the remaining sections are as follows. Section 2 recalls several definitions from [5] and [10]. Intersection formulas are analyzed in Section 3. Section 4 presents formulas for computing the subdifferential and the singular subdifferential of the marginal 
functions of convex optimization problems under inclusion constraints on Banach spaces. Section 5 compares our results with some known results. Several conclusions are given in the final section.

\section{Preliminaries}

Let $X$ and $Y$ be Banach spaces whose duals are denoted, respectively, by $X^{*}$ and $Y^{*}$. We use $\mathbb{B}(x, r)$ to denote the open ball centered at $x$ with radius $r>0$. Let $\mathbb{B}_{X}$ (resp. $\mathbb{B}_{X^{*}}$ ) denote the closed unit ball of $X$ (resp. $X^{*}$ ) centered at the origin. The closure and the interior of a set $A$ are denoted by $\operatorname{cl} A$ and $\operatorname{int} A$, respectively.

Let $\varphi: X \rightarrow \overline{\mathbb{R}}$ be an extended real-valued function, where $\overline{\mathbb{R}}:=[-\infty,+\infty]=\mathbb{R} \cup\{+\infty\} \cup$ $\{-\infty\}$. One says that $\varphi$ is proper if the domain

$$
\operatorname{dom} \varphi:=\{x \in X: \varphi(x)<+\infty\}
$$

is nonempty, and if $\varphi(x)>-\infty$ for all $x \in X$. The function $\varphi$ is convex if and only if its epigraph

$$
\operatorname{epi} \varphi:=\{(x, \alpha) \in X \times \mathbb{R}: \alpha \geq \varphi(x)\}
$$

is a convex subset of $X \times \mathbb{R}$. If epi $\varphi$ is a closed subset of $X \times \mathbb{R}, \varphi$ is said to be a closed function. Denoting the set of all the neighborhoods of $x$ by $\mathcal{N}(x)$, one says that $\varphi$ is lower semicontinuous (l.s.c.) at $x \in X$ if for every $\varepsilon>0$ there exists $U \in \mathcal{N}(x)$ such that $\varphi\left(x^{\prime}\right) \geq \varphi(x)-\varepsilon$ for any $x^{\prime} \in U$. If $\varphi$ is l.s.c. at every $x \in X, \varphi$ is said to be l.s.c. on $X$. It is convenient to denote the set of all proper l.s.c. convex functions on $X$ by $\Gamma_{0}(X)$.

Let $\varphi$ be an extended-real valued convex function on $X$ and $\bar{x} \in \operatorname{dom} \varphi$. The subdifferential of $\varphi$ at $\bar{x}$ is the set

$$
\partial \varphi(\bar{x})=\left\{x^{*} \in X^{*}:\left\langle x^{*}, x-\bar{x}\right\rangle \leq \varphi(x)-\varphi(\bar{x}), \quad \forall x \in X\right\} .
$$

If $f \in \Gamma_{0}(X)$, then $\partial \varphi(\bar{x})$ is nonempty for every $\bar{x} \in \operatorname{int}(\operatorname{dom}) \varphi$ (see [15]). 
For a convex set $\Omega \subset X$, the normal cone of $\Omega$ at $\bar{x} \in \Omega$ is given by

$$
N(\bar{x} ; \Omega)=\left\{x^{*} \in X^{*}:\left\langle x^{*}, x-\bar{x}\right\rangle \leq 0, \quad \forall x \in \Omega\right\} .
$$

The singular subdifferential of a convex function $\varphi$ at $\bar{x} \in \operatorname{dom} \varphi$ is defined by

$$
\partial^{\infty} \varphi(\bar{x})=\left\{x^{*} \in X^{*}:\left(x^{*}, 0\right) \in N((\bar{x}, \varphi(\bar{x})) ; \text { epi } \varphi)\right\} .
$$

For any $\bar{x} \notin \operatorname{dom} \varphi$, we put $\partial \varphi(\bar{x})=\emptyset$ and $\partial^{\infty} \varphi(\bar{x})=\emptyset$.

It is not difficult to show that $x^{*} \in \partial \varphi(\bar{x})$ if and only if $\left\langle x^{*}, x-\bar{x}\right\rangle-(\alpha-\varphi(\bar{x})) \leq 0$ for all $(x, \alpha) \in$ epi $\varphi$ or, equivalently, $\left(x^{*},-1\right) \in N((\bar{x}, \varphi(\bar{x}))$; epi $\varphi)$. Interestingly, for any convex function $\varphi$, one has $\partial^{\infty} \varphi(x)=N(x$; dom $\varphi)$; see e.g., [2].

Let $F: X \rightrightarrows Y$ be a multivalued function. The graph and the domain of $F$ are defined by

$$
\operatorname{Gr} F:=\{(x, y) \in X \times Y: y \in F(x)\} \text { and } \operatorname{dom} F:=\{x \in X: F(x) \neq \emptyset\} .
$$

One says that a multivalued function $F$ is closed (resp., convex) if Gr $F$ is closed (resp., convex). In addition, if $\operatorname{dom} F \neq \emptyset, F$ is called proper. The product space $X \times Y$ will be endowed with the norm $\|(x, y)\|=\|x\|+\|y\|$ for any $(x, y) \in X \times Y$.

The coderivative $D^{*} F(\bar{x}, \bar{y}): Y^{*} \rightrightarrows X^{*}$ of a convex multifunction $F$ between $X$ and $Y$ at $(\bar{x}, \bar{y}) \in \operatorname{Gr} F$ is the multivalued function defined by

$$
D^{*} F(\bar{x}, \bar{y})\left(y^{*}\right):=\left\{x^{*} \in X^{*}:\left(x^{*},-y^{*}\right) \in N((\bar{x}, \bar{y}) ; \operatorname{Gr} F)\right\}, \forall y^{*} \in Y^{*}
$$

If $(\bar{x}, \bar{y}) \notin \operatorname{Gr} F$, one puts $D^{*} F(\bar{x}, \bar{y})\left(y^{*}\right)=\emptyset$ for any $y^{*} \in Y^{*}$.

Let $C$ be a nonempty subset of $X$. The distance function of $C$ is defined by

$$
d(x, C)=\inf _{u \in C}\|x-u\|
$$

Clearly, the distance function $d(\cdot, C)$ is Lipschitz continuous with modulus one, and convex provided that $C$ is convex. It is obvious that for any convex set $\Omega \subset X$ containing $\bar{x}$, we have

$$
N(\bar{x} ; \Omega)=N(\bar{x} ; \operatorname{cl} \Omega) \text { and } \partial d(\bar{x}, \Omega)=\partial d(\bar{x}, \operatorname{cl} \Omega)
$$


We will need some properties of the subdifferential of $d(\cdot, C)$. Assertion (i) of the following proposition has been observed in different places, e.g., [5, Example 2.130] and [8, Theorem 1]. Assertion (ii) is well known when $C$ is convex as it corresponds to [9, Proposition 11]. We present a direct proof for the reader's convenience.

Proposition 2.1. Let $C$ be a nonempty convex subset of $X$. Then for every $x \in C$, one has

(i) $\partial d(x, C)=\mathbb{B}_{X^{*}} \cap N(x ; C)$, where $\mathbb{B}_{X^{*}}$ is the closed unit ball in $X^{*}$;

(ii) $N(x ; C)=\bigcup_{\lambda>0} \lambda \partial d(x, C)$.

Proof. We only need to prove assertion (i) beacause (ii) immediately follows from the first one. Indeed, by the definition of the subdifferential, one has $x^{*} \in \partial d(x, C)$ if and only if

$$
\left\langle x^{*}, u-x\right\rangle \leq d(u, C)-d(x, C), \forall u \in X \text {. }
$$

Since $d(\cdot, C)$ is Lipschitz continuous modulus one, from (4) one has

$$
\left\langle x^{*}, u-x\right\rangle \leq d(u, C)-d(x, C) \leq\|u-x\|, \forall u \in X
$$

It follows that $\left\|x^{*}\right\| \leq 1$. Hence $\partial d(x, C) \subset \mathbb{B}_{X^{*}}$. Moreover, for all $u \in C$, from (4) we get $\left\langle x^{*}, u-x\right\rangle \leq 0$. In other words, $x^{*} \in N(x ; C)$. Therefore $x^{*} \in \mathbb{B}_{X^{*}} \cap N(x ; C)$.

Conversely, suppose that $x^{*}$ belongs to $\mathbb{B}_{X^{*}} \cap N(x ; C)$. For any $u \in X$ and $\varepsilon>0$, we can find $u^{\prime} \in C$ such that $d(u, C) \geq\left\|u-u^{\prime}\right\|-\varepsilon$. As $\left\|x^{*}\right\| \leq 1$ and $x^{*} \in N(x ; C)$, the latter implies that

$$
\begin{aligned}
d(u, C) \geq\left\langle x^{*}, u-u^{\prime}\right\rangle-\varepsilon & =\left\langle x^{*}, u-x\right\rangle+\left\langle x^{*}, x-u^{\prime}\right\rangle-\varepsilon \\
& \geq\left\langle x^{*}, u-x\right\rangle-\varepsilon .
\end{aligned}
$$

Since $\varepsilon>0$ is arbitrary and $x \in C$, we obtain

$$
d(u, C) \geq\left\langle x^{*}, u-x\right\rangle=\left\langle x^{*}, u-x\right\rangle+d(x, C) \quad \forall u \in X
$$

which shows that $x^{*} \in \partial d(x, C)$. 


\section{$3 \quad$ Intersection formulas}

Let $C$ and $D$ be subsets of $X$ with $x_{0} \in C \cap D$. One says that $C$ and $D$ satisfy the intersection formula at $x_{0}$ if there exist two real numbers $a>0$ and $r>0$ such that inequality (1) is satisfied. In the case where $C$ and $D$ are closed and convex, we obtain (see [4]) the following characterization.

Proposition 3.1. Let $C$ and $D$ be closed and convex subset of $X$ and $a>0$. Then the following assertions are equivalent

1. There exists $r>0$ satisfying relation (1),

2. There exists $s>0$ such that

$$
\partial d(x, C \cap D) \subset a \partial d(x, C)+a \partial d(x, D) \quad \forall x \in C \cap D \cap \mathbb{B}\left(x_{0}, s\right) .
$$

Proof. It is given for completeness. The implication $1 . \Rightarrow 2$. is obvious. So let us establish

the reverse one. Let $u \in \mathbb{B}\left(x_{0}, \frac{s}{2}\right) \backslash C \cap D$ and $\left.\varepsilon \in\right] 0, \frac{\sqrt{s}}{4}[$. Then, by Lemma 2.1 in [4], there exist $u_{\varepsilon} \in C \cap D, x_{\varepsilon}^{*} \in X^{*}$ and $b_{\varepsilon}^{*} \in \mathbb{B}^{*}$ such that

i) $\left\|u_{\varepsilon}-u\right\| \leq d(u, C \cap D)+\varepsilon^{2}$,

ii) $x_{\varepsilon}^{*}+\varepsilon b_{\varepsilon}^{*} \in(1+\varepsilon) \partial d\left(u_{\varepsilon}, C \cap D\right)$,

iii) $\left\langle x_{\varepsilon}^{*}, u-u_{\varepsilon}\right\rangle=\left\|u_{\varepsilon}-u\right\|$.

Note that $u_{\varepsilon} \in C \cap D \cap \mathbb{B}\left(x_{0}, s\right)$. By 2. and $\left.i i\right)$, we have

$$
\begin{aligned}
x_{\varepsilon}^{*}+\varepsilon b_{\varepsilon}^{*} & \in a(1+\varepsilon)\left[\partial d\left(u_{\varepsilon}, C\right)+\partial d\left(u_{\varepsilon}, D\right)\right] \\
& =a(1+\varepsilon) \partial(d(\cdot, C)+d(\cdot, D))\left(u_{\varepsilon}\right)
\end{aligned}
$$

and hence

$$
\left\langle x_{\varepsilon}^{*}+\varepsilon b_{\varepsilon}^{*}, u-u_{\varepsilon}\right\rangle \leq a(1+\varepsilon)(d(u, C)+d(u, D)) .
$$


Using $i i i)$, we get

$$
\left\|u-u_{\varepsilon}\right\|(1-\varepsilon) \leq a(1+\varepsilon)(d(u, C)+d(u, D))\left(\text { because }\left\|b_{\varepsilon}^{*}\right\| \leq 1\right)
$$

which in turns implies that

$$
d(u, C \cap D)(1-\varepsilon) \leq a(1+\varepsilon)(d(u, C)+d(u, D))
$$

and as $\varepsilon$ is arbitrary, we obtain the desired result by putting $r=\frac{s}{2}$.

Following Borwein and Strojwas [7], we call a set $C \subset X$ compactly epi-Lipschitzian (CEL) at $x_{0} \in C$ if there exist a norm-compact set $K$ and a scalar $r>0$ such that

$$
C \cap\left(x_{0}+r \mathbb{B}_{X}\right)+\tau r \mathbb{B}_{X} \subset C-\tau K \quad \forall \tau \in[0, r]
$$

This class includes all finite dimensional sets. It is worth to note that in infinite dimensional spaces, there is no relationship between compact sets and CEL sets (because these later are never compact in infinite dimensional spaces). This also implies that the CEL sets are useful only in infinite dimensional spaces; see ([6], [17]).

In [13], it is shown that a boundary condition ensures the intersection formula. More precisely, one has

Proposition 3.2. Let $C$ and $D$ be closed convex subsets of $X$ and $x_{0} \in C \cap D$. Suppose that one of the sets $C$ or $D$ is $C E L$ at $x_{0}$ and

$$
\operatorname{bd}\left(N\left(x_{0} ; C\right)\right) \cap\left[-\operatorname{bd}\left(N\left(x_{0} ; D\right)\right)\right]=\{0\}
$$

Then there exist $r>0$ and $a>0$ satisfying relation (1). Here "bd(A)" denotes the boundary of the set $A \subset X^{*}$ with respect to the norm on $X^{*}$.

Remark 3.1. Note that the reverse implication is not true. We mean that relation (1) does not implies (6). To see this, take

$$
C:=\left\{\left(x_{1}, x_{2}\right) \in \mathbb{R}^{2}: x_{1} \leq 0, x_{2} \leq 0, x_{2} \leq x_{1}\right\}
$$

$D=\mathbb{R}_{+}^{2}$ and $x_{0}=(0,0)$. It can be verified that relation (1) is valid for $a=1$ and for any $r>0$, meanwhile

$$
\operatorname{bd} N\left(x_{0} ; C\right) \cap\left[-\operatorname{bd} N\left(x_{0} ; D\right)\right]=\mathbb{R}_{+} \times\{0\}
$$




\section{Main results}

We define the marginal function (also called value function) $m: X \rightarrow \overline{\mathbb{R}}$ by

$$
m(x):=\inf \{f(x, y): y \in F(x)\}
$$

where $f$ is an extended-real-valued convex lower semicontinuous function on $X \times Y$ and $F$ is a multivalued function from $X$ to $Y$ with closed graph. By the convention inf $\emptyset=+\infty$, we have $m(x)=+\infty$ for all $x \notin \operatorname{dom} F$. Corresponding to each data pair $F, f$ we have one optimization problem depending on a parameter $x$ :

$$
\min \{f(x, y): y \in F(x)\}
$$

For every $x \in \operatorname{dom} m$, we consider the set of minimizers

$$
M(x)=\{y \in F(x): m(x)=f(x, y)\}
$$

We are looking for formulas to estimate/compute subdifferentials of $m$.

The following proposition gives the continuity of $m$ under some conditions.

Proposition 4.1. The marginal function $m(\cdot)$ is convex. Moreover if $m$ is finite at $x_{0}$, $m(\cdot)$ is continuous at $x_{0}$ if and only if for every $x$ in a neighborhood of $x_{0}, m(x)<+\infty$, i.e., $x_{0} \in \operatorname{int}(\operatorname{dom} m)$.

Proof. We consider the unconstrained problem

$$
\min \{\varphi(x, y): y \in Y\}
$$

with the objective function $\varphi(x, y)$ being $\varphi(x, y)=f(x, y)+\delta_{\mathrm{Gr} F}(x, y)$. Obviously, the marginal function of problem (10) is also the function $m$ given in (7). First, since $F$ is a convex multivalued function, it follows that $\operatorname{Gr} F$ is a convex set. So $\delta_{\mathrm{Gr} F}(\cdot)$ is a convex function. Combining this with the convexity of $f$ yields the function $\varphi$ is convex. By [5, Proposition 2.143], the marginal function $m$ is convex. Moreover, since $F$ is a proper closed multivalued function, one has $\delta_{\mathrm{Gr} F}(\cdot)$ is a proper lower semicontinuous function. Thus $\varphi$ is proper lower semicontinuous. Therefore the second part is obtained by applying [5, Proposition 2.152]. 
Put

$$
C_{1}=\text { epi } f \text { and } C_{2}=\operatorname{Gr} F \times \mathbb{R} \text {. }
$$

We need some auxiliary results.

Lemma 4.1. Let $y_{0} \in M\left(x_{0}\right)$. Then, we have $\left(x^{*}, \beta\right) \in N\left(\left(x_{0}, m\left(x_{0}\right)\right)\right.$; epi $\left.m\right)$ if and only if

$$
\left(x^{*}, 0, \beta\right) \in N\left(\left(x_{0}, y_{0}, f\left(x_{0}, y_{0}\right)\right) ; C_{1} \cap C_{2}\right)
$$

Proof. Let $\left(x^{*}, \beta\right) \in N\left(\left(x_{0}, m\left(x_{0}\right)\right)\right.$, epi $\left.m\right)$. Then,

$$
\left\langle x^{*}, x-x_{0}\right\rangle+\beta\left(r-m\left(x_{0}\right)\right) \leq 0, \forall(x, r) \in \text { epi } m
$$

because $\left(x_{0}, \lambda+m\left(x_{0}\right)\right) \in$ epi $m$ for every $\lambda \geq 0$. So $\beta$ must be negative number. Keeping in mind that $y_{0} \in M\left(x_{0}\right)$, from (11) one has

$$
\begin{aligned}
\left\langle x^{*}, x-x_{0}\right\rangle & \leq-\beta\left(r-m\left(x_{0}\right)\right), \forall(x, r) \in \text { epi } m \\
& \leq-\beta\left(m(x)-f\left(x_{0}, y_{0}\right)\right), \forall x \in \operatorname{dom} m \\
& \leq-\beta\left(f(x, y)-f\left(x_{0}, y_{0}\right)\right), \forall(x, y) \in \operatorname{dom} f \text { and }(x, y) \in \operatorname{Gr} F \\
& \leq-\beta\left(r-f\left(x_{0}, y_{0}\right)\right), \forall(x, y, r) \in \operatorname{epi} f \text { and }(x, y) \in \operatorname{Gr} F
\end{aligned}
$$

or, equivalently,

$$
\left\langle\left(x^{*}, 0\right),(x, y)-\left(x_{0}, y_{0}\right)\right\rangle+\beta\left(r-f\left(x_{0}, y_{0}\right)\right) \leq 0, \forall(x, y, r) \in \text { epi } f \text { and }(x, y) \in \text { Gr } F \text {. }
$$

So $\left(x^{*}, 0, \beta\right) \in N\left(\left(x_{0}, y_{0}, f\left(x_{0}, y_{0}\right)\right) ; C_{1} \cap C_{2}\right)$, with $C_{1}=$ epi $f$ and $C_{2}=\operatorname{Gr} F \times \mathbb{R}$.

Conversely, take $\left(x^{*}, 0, \beta\right) \in N\left(\left(x_{0}, y_{0}, f\left(x_{0}, y_{0}\right)\right) ; C_{1} \cap C_{2}\right)$. By the definition of the normal cone, we have

$$
\left\langle x^{*}, x-x_{0}\right\rangle+\beta\left(r-f\left(x_{0}, y_{0}\right)\right) \leq 0, \forall(x, y, r) \in C_{1} \cap C_{2} .
$$

Similarly, one has $\beta \leq 0$. By the definition of the infimum, for all $\varepsilon \geq 0$, we can find $y \in F(x)$ such that $m(x) \geq f(x, y)-\varepsilon$. Put $r=\varepsilon+m(x)$, we have $(x, y, r) \in C_{1} \cap C_{2}$. From (12), we obtain

$$
\left\langle x^{*}, x-x_{0}\right\rangle \leq-\beta\left(\varepsilon+m(x)-f\left(x_{0}, y_{0}\right)\right)=-\beta\left(\varepsilon+m(x)-m\left(x_{0}\right)\right), \forall x \in \operatorname{dom} m .
$$


The last inequality implies that

$$
\begin{aligned}
\left\langle x^{*}, x-x_{0}\right\rangle & \leq-\beta\left(m(x)-m\left(x_{0}\right)\right), \forall x \in \operatorname{dom} m \\
& \leq-\beta\left(r-m\left(x_{0}\right)\right), \forall(x, r) \in \operatorname{epi} m .
\end{aligned}
$$

In other words, $\left(x^{*}, \beta\right) \in N\left(\left(x_{0}, m\left(x_{0}\right)\right)\right.$; epi $\left.m\right)$.

Lemma 4.2. Let $y_{0} \in M\left(x_{0}\right)$. Then one has

$$
\left(x^{*}, \beta\right) \in \partial d\left(\left(x_{0}, m\left(x_{0}\right)\right), \text { epi } m\right)
$$

if and only if

$$
\left(x^{*}, 0, \beta\right) \in \partial d\left(\left(x_{0}, y_{0}, f\left(x_{0}, y_{0}\right)\right), C_{1} \cap C_{2}\right) .
$$

Proof. According to Propostion 2.1(i), one has

$$
\partial d\left(\left(x_{0}, m\left(x_{0}\right)\right) \text {, epi } m\right)=\mathbb{B}_{X^{*} \times \mathbb{R}} \cap N\left(\left(x_{0}, m\left(x_{0}\right)\right) ; \text { epi } m\right) .
$$

Then, $\left(x^{*}, \beta\right) \in \partial d\left(\left(x_{0}, m\left(x_{0}\right)\right)\right.$; epi $\left.m\right)$ if and only if $\left\|\left(x^{*}, \beta\right)\right\| \leq 1$ and

$$
\left(x^{*}, \beta\right) \in N\left(\left(x_{0}, m\left(x_{0}\right)\right) ; \text { epi } m\right) .
$$

By Lemma $4.1,\left(x^{*}, \beta\right) \in N\left(\left(x_{0}, m\left(x_{0}\right)\right)\right.$; epi $\left.m\right)$ if and only if

$$
\left(x^{*}, 0, \beta\right) \in N\left(\left(x_{0}, y_{0}, f\left(x_{0}, y_{0}\right)\right) ; C_{1} \cap C_{2}\right) .
$$

Combining with $\left\|\left(x^{*}, 0, \beta\right)\right\| \leq 1$, we obtain $\left(x^{*}, \beta\right) \in \partial d\left(\left(x_{0}, m\left(x_{0}\right)\right)\right.$, epi $\left.m\right)$ if and only if $\left(x^{*}, 0, \beta\right) \in \partial d\left(\left(x_{0}, y_{0}, f\left(x_{0}, y_{0}\right)\right), C_{1} \cap C_{2}\right)$.

The first main result of this section reads as follows.

Theorem 4.1. Suppose that $C_{1}$ and $C_{2}$ satisfy the intersection formula at $\left(x_{0}, y_{0}, f\left(x_{0}, y_{0}\right)\right)$, with $y_{0} \in M\left(x_{0}\right)$. Then

$$
\partial m\left(x_{0}\right)=\bigcup_{\left(x^{*}, y^{*}\right) \in \partial f\left(x_{0}, y_{0}\right)}\left\{x^{*}+D^{*} F\left(x_{0}, y_{0}\right)\left(y^{*}\right)\right\} .
$$


Proof. Pick $u^{*}$ from the right hand side of (13), then there exists $\left(x^{*}, y^{*}\right) \in \partial f\left(x_{0}, y_{0}\right)$ such that

$$
u^{*} \in x^{*}+D^{*} F\left(x_{0}, y_{0}\right)\left(y^{*}\right) .
$$

It gives us $\left(u^{*}-x^{*},-y^{*}\right) \in N\left(\left(x_{0}, y_{0}\right) ; \operatorname{Gr} F\right)$. By the definition of the normal cone, we have

$$
\left\langle u^{*}-x^{*}, x-x_{0}\right\rangle-\left\langle y^{*}, y-y_{0}\right\rangle \leq 0, \forall(x, y) \in \operatorname{Gr} F,
$$

which shows that whenever $y \in F(x)$, we have

$$
\begin{aligned}
\left\langle u^{*}, x-x_{0}\right\rangle & \leq\left\langle x^{*}, x-x_{0}\right\rangle+\left\langle y^{*}, y-y_{0}\right\rangle \\
& \leq f(x, y)-f\left(x_{0}, y_{0}\right) \\
& \leq f(x, y)-m\left(x_{0}\right) .
\end{aligned}
$$

The latter implies

$$
\left\langle u^{*}, x-x_{0}\right\rangle \leq \inf _{y \in F(x)} f(x, y)-m\left(x_{0}\right)=m(x)-m\left(x_{0}\right), \forall y \in F(x) .
$$

Therefore $u^{*} \in \partial m\left(x_{0}\right)$.

To establish the opposite inclusion, we note that

$$
u^{*} \in \partial m\left(x_{0}\right) \Leftrightarrow\left(u^{*},-1\right) \in N\left(\left(x_{0}, m\left(x_{0}\right)\right) ; \text { epi } m\right) .
$$

According to Proposition 2.1(ii), one gets

$$
N\left(\left(x_{0}, m\left(x_{0}\right)\right) ; \text { epi } m\right)=\bigcup_{\lambda \geq 0} \lambda \partial d\left(\left(x_{0}, m\left(x_{0}\right)\right) ; \text { epi } m\right) .
$$

In other words, there exist $\lambda>0$ and $\left(x^{*}, \beta\right) \in \partial d\left(\left(x_{0}, m\left(x_{0}\right)\right)\right.$; epi $\left.m\right)$ such that $u^{*}=\lambda x^{*}$ and $\lambda \beta=-1$. By Lemma 4.2, the latter is equivalent to

$$
\left(x^{*}, 0, \beta\right) \in \partial d\left(\left(x_{0}, y_{0}, f\left(x_{0}, y_{0}\right)\right), C_{1} \cap C_{2}\right) .
$$

Our assumptions guarantee the convexity and the closedness of the sets $C_{1}=\operatorname{epi} f$ and $C_{2}=\operatorname{Gr} F \times \mathbb{R}$. Since $C_{1}$ and $C_{2}$ satisfy the intersection formula at $\left(x_{0}, y_{0}, f\left(x_{0}, y_{0}\right)\right)$, 
Proposition 3.1 ensures the existence of $a>0$ satisfying

$$
\partial d\left(\left(x_{0}, y_{0}, f\left(x_{0}, y_{0}\right)\right), C_{1} \cap C_{2}\right) \subset a\left[\partial d\left(\left(x_{0}, y_{0}, f\left(x_{0}, y_{0}\right)\right), C_{1}\right)+\partial d\left(\left(x_{0}, y_{0}, f\left(x_{0}, y_{0}\right)\right), C_{2}\right)\right]
$$

Therefore, from (14) and (15), we can find a vector $\left(z^{*}, y^{*}, \gamma\right) \in \partial d\left(\left(x_{0}, y_{0}, f\left(x_{0}, y_{0}\right)\right)\right.$, epi $\left.f\right)$, and $\left(v^{*}, p^{*}\right) \in \partial d\left(\left(x_{0}, y_{0}\right), \operatorname{Gr} F\right)$ satisfying

$$
\begin{aligned}
& x^{*}=a\left(z^{*}+v^{*}\right), \\
& 0=a\left(y^{*}+p^{*}\right),
\end{aligned}
$$

and $\beta=a \gamma$. Hence

$$
\begin{aligned}
& u^{*}=\lambda x^{*}=\lambda a\left(z^{*}+v^{*}\right), \\
& -1=\lambda \beta=a \lambda \gamma
\end{aligned}
$$

and $\lambda a y^{*}=-\lambda a p^{*}$. Thus $\left(\lambda a z^{*}, \lambda a y^{*}\right) \in \partial f\left(x_{0}, y_{0}\right)$, and

$$
u^{*} \in \lambda a z^{*}+D F\left(x_{0}, y_{0}\right)\left(\lambda a y^{*}\right) .
$$

By Proposition 3.2, the next corollary follows directly from Theorem 4.1.

Corollary 4.1. Let $y_{0} \in M\left(x_{0}\right)$. Suppose that one of the following conditions is satisfied (i) epi $f$ is CEL at $\left(x_{0}, y_{0}, f\left(x_{0}, y_{0}\right)\right)$ and

$$
N\left(\left(x_{0}, y_{0}, f\left(x_{0}, y_{0}\right)\right) ; C_{1}\right) \cap\left[-N\left(\left(x_{0}, y_{0}, f\left(x_{0}, y_{0}\right)\right) ; C_{2}\right)\right]=\left\{\left(0_{X^{*}}, 0_{Y^{*}}, 0_{\mathbb{R}}\right)\right\}
$$

(ii) $\operatorname{Gr} F \times \mathbb{R}$ is CEL at $\left(x_{0}, y_{0}, f\left(x_{0}, y_{0}\right)\right)$ and (16) holds.

Then the conclusion of Theorem 4.1 is valid.

The singular subdifferential of $m$ can be computed as follows.

Theorem 4.2. Let $y_{0} \in M\left(x_{0}\right)$. Suppose that one of the following conditions is satisfied (i) dom $f$ is CEL at $\left(x_{0}, y_{0}, f\left(x_{0}, y_{0}\right)\right)$ and

$$
N\left(\left(x_{0}, y_{0}\right) ; \operatorname{dom} f\right) \cap\left[-N\left(\left(x_{0}, y_{0}\right) ; \operatorname{Gr} F\right)\right]=\left\{\left(0_{X^{*}}, 0_{Y^{*}}\right)\right\}
$$


(ii) Gr $F \times \mathbb{R}$ is CEL at $\left(x_{0}, y_{0}, f\left(x_{0}, y_{0}\right)\right)$ and (17) holds. Then

$$
\partial^{\infty} m\left(x_{0}\right)=\bigcup_{\left(x^{*}, y^{*}\right) \in \partial^{\infty} f\left(x_{0}, y_{0}\right)}\left\{x^{*}+D^{*} F\left(x_{0}, y_{0}\right)\left(y^{*}\right)\right\} .
$$

Proof. We first observe that $x \in \operatorname{dom} m$ if and only if

$$
m(x)=\inf \{f(x, y): y \in F(x)\}<+\infty .
$$

As the strict inequality holds if and only if there exists $y \in F(x)$ with $(x, y) \in \operatorname{dom} F$, we have

$$
\delta_{\operatorname{dom} m}(x)=\inf \left\{\delta_{\operatorname{dom} f}(x, y): y \in F(x)\right\} .
$$

Recall that $\delta_{C}$ is the indicator function of the set $C$, i.e., $\delta_{C}(x)=0$ if $x \in C$ and $+\infty$, otherwise. On one hand, epi $\delta_{\operatorname{dom} f}=\operatorname{dom} f \times[0,+\infty)$. If the condition (i) is satisfied, then epi $\delta_{\operatorname{dom} f}$ is CEL at $\left(x_{0}, y_{0}, f\left(x_{0}, y_{0}\right)\right)$. On the other hand, (16) yields

$$
\begin{aligned}
N\left(\left(x_{0}, y_{0}, \delta_{\operatorname{dom} f}\left(x_{0}, y_{0}\right)\right) ; \operatorname{epi} \delta_{\operatorname{dom} f}\right) \cap\left[-N\left(\left(x_{0}, y_{0}, \delta_{\operatorname{dom} f}\left(x_{0}, y_{0}\right)\right) ; \operatorname{Gr} F \times \mathbb{R}\right)\right] & =\left\{0_{X^{*}}, 0_{Y^{*}}, 0_{\mathbb{R}}\right\} .
\end{aligned}
$$

Since $f$ is convex, it follows that dom $f$ is convex. Moreover, as $f$ is lower semicontinuous, it follows that epi $f$ and $\operatorname{dom} f$ are closed. Thus $\delta_{\operatorname{dom} f}($.$) is a lower semincontinuous$ function. So all assumptions of Corollary 4.1 are satisfied. Thanks to Corollary 4.1, we have

$$
\partial \delta_{\operatorname{dom} m}\left(x_{0}\right)=\bigcup_{\left(x^{*}, y^{*}\right) \in \partial \delta_{\operatorname{dom} f}\left(x_{0}, y_{0}\right)}\left\{x^{*}+D^{*} F\left(x_{0}, y_{0}\right)\left(y^{*}\right)\right\} .
$$

As $\partial \delta_{\operatorname{dom} m}\left(x_{0}\right)=\partial^{\infty} m\left(x_{0}\right)$ and $\partial \delta_{\operatorname{dom} f}\left(x_{0}, y_{0}\right)=\partial^{\infty} f\left(x_{0}, y_{0}\right)$. So, we obtain (18) from (19).

\section{Comparisons with some known results}

By a different approach, the authors in [1] and [2] have studied a problem similar to that considered in this paper. Namely, in our notation, by using some suitable sum rules for convex subdifferential, the authors have proved the following theorems. 
Theorem 5.1. (see [2, Theorem 4.2]) Let $X$ and $Y$ be Hausdorff locally convex topological vector spaces. Consider the problem (8) with $F: X \rightrightarrows Y$ being a convex multivalued function and $f: X \times Y \rightarrow \overline{\mathbb{R}}$ being a proper convex function. If at least one of the following regularity conditions is satisfied:

(a) $\operatorname{int}(\operatorname{Gr} F) \cap \operatorname{dom} f \neq \emptyset$,

(b) $f$ is continuous at a point $\left(x^{0}, y^{0}\right) \in \mathrm{Gr} F$,

then, for any $\bar{x} \in \operatorname{dom} m$, with $m(\bar{x}) \neq-\infty$, and for any $\bar{y} \in M(\bar{x})$ we have

$$
\partial m(\bar{x})=\bigcup_{\left(x^{*}, y^{*}\right) \in \partial f(\bar{x}, \bar{y})}\left\{x^{*}+D^{*} F(\bar{x}, \bar{y})\left(y^{*}\right)\right\}
$$

and

$$
\partial^{\infty} m(\bar{x})=\bigcup_{\left(x^{*}, y^{*}\right) \in \partial^{\infty} f(\bar{x}, \bar{y})}\left\{x^{*}+D^{*} F(\bar{x}, \bar{y})\left(y^{*}\right)\right\},
$$

where $m(\cdot)$ and $M(\cdot)$ are given respectively by $(7)$ and $(9)$.

Theorem 5.2. (see [1, Theorems 3.3 and 3.4]) Consider the problem (8) with $F: X \rightrightarrows Y$ being a convex multifunction whose graph is closed, and $f: X \times Y \rightarrow \overline{\mathbb{R}}$ a proper, closed, convex function. If

$$
(0,0) \in \operatorname{int}(\operatorname{dom} f-\operatorname{Gr} F)
$$

is satisfied, then for every $\bar{x} \in \operatorname{dom} m$ with $m(\bar{x}) \neq-\infty$, and for any $\bar{y} \in M(\bar{x})$, we have

$$
\partial m(\bar{x})=\bigcup_{\left(x^{*}, y^{*}\right) \in \partial f(\bar{x}, \bar{y})}\left\{x^{*}+D^{*} F(\bar{x}, \bar{y})\left(y^{*}\right)\right\} .
$$

Suppose further that the set $\operatorname{dom} f$ is closed. Then

$$
\partial^{\infty} m(\bar{x})=\bigcup_{\left(x^{*}, y^{*}\right) \in \partial^{\infty} f(\bar{x}, \bar{y})}\left\{x^{*}+D^{*} F(\bar{x}, \bar{y})\left(y^{*}\right)\right\} .
$$

In [1], the authors have shown the relationship between regularity conditions. Namely, the condition (20) is really weaker than both regularity assumptions (a) and (b) in Theorem 5.1 (see [1, Section 4]). 
We now give an analysis of regularity conditions. Let $X$ be a finite dimensional space. It is a convenient way to consider the following problem

$$
\min \{f(x): x \in C\}
$$

where $f: X \rightarrow \mathbb{R} \cup\{+\infty\}$ is a closed, convex function and $C \subset X$ is a closed, convex set.

First, we recall that all subsets of $X$ are CEL (see [7, Proposition 2.4]) and every convex function $f$ defined on a finite dimensional space is continuous on $\operatorname{int}(\operatorname{dom} f)$ (see, e.g., [5, Corollary 2.109]). We have the following proposition.

Proposition 5.1. The regularity condition

$$
0 \in(\operatorname{dom} f-C)
$$

implies

$$
N\left(\left(x_{0}, f\left(x_{0}\right)\right) ; \text { epi } f\right) \cap\left[-N\left(\left(x_{0}, f\left(x_{0}\right)\right) ; C \times \mathbb{R}\right)\right]=\{(0,0)\}
$$

Proof. Take an arbitrary $\left(x^{*}, \beta\right) \in N\left(\left(x_{0}, f\left(x_{0}\right)\right)\right.$; epi $\left.f\right) \cap\left[-N\left(\left(x_{0}, f\left(x_{0}\right)\right) ; C \times \mathbb{R}\right)\right]$. We first observe that for every $x_{0} \in C$ with $\left(x_{0}, f\left(x_{0}\right)\right) \in$ epi $f$,

$$
N\left(\left(x_{0}, f\left(x_{0}\right)\right) ; C \times \mathbb{R}\right)=N\left(x_{0} ; C\right) \times N\left(f\left(x_{0}\right) ; \mathbb{R}\right)=N\left(x_{0} ; C\right) \times\{0\} .
$$

Thus $\beta=0$. Moreover, we have

$$
\left\langle x^{*}, x-x_{0}\right\rangle+0 .\left(r-f\left(x_{0}\right)\right) \leq 0, \forall(x, r) \in \text { epi } f
$$

and

$$
\left\langle-x^{*}, x-x_{0}\right\rangle \leq 0, \forall x \in C
$$

On one hand, from (24) one has $\left\langle x^{*}, y-x_{0}\right\rangle \leq 0, \forall y \in \operatorname{dom} f$. Combining this with (25), we obtain $\left\langle x^{*}, y-x\right\rangle \leq 0, \forall y \in \operatorname{dom} f, x \in C$, or

$$
\left\langle x^{*}, h\right\rangle \leq 0, \forall h \in(\operatorname{dom} f-C)
$$


On the other hand, by the assumption $0 \in(\operatorname{dom} f-C)$, we can find a neighborhood $U$ of 0 such that $\left\langle x^{*}, h\right\rangle \leq 0$, for all $h \in U$. The later yields $x^{*}=0$. Combining this with $\beta=0$, we obtain $\left(x^{*}, \beta\right)=(0,0)$.

Let us consider an example to show that the regularity condition (22) is really stronger than (23).

Example 5.1. Let $X=\mathbb{R}^{2}$ and $C=\left\{(x, y) \in \mathbb{R}^{2}: y=x, y \geq 0\right\}$. Consider the function $f$ given by

$$
f(x, y)= \begin{cases}0, & \text { if } y=0 \\ +\infty, & \text { if } y \neq 0 .\end{cases}
$$

For $x_{0}=y_{0}=0$. On one hand, one has $N\left(\left(x_{0}, y_{0}, f\left(x_{0} . y_{0}\right)\right) ; C \times \mathbb{R}\right)=\left\{\left(0_{\mathbb{R}^{2}}, 0\right)\right\}$. So (23) is satisfied. On the other hand $\operatorname{dom} f=\mathbb{R} \times\{0\}$. Then $(0,0) \notin(\operatorname{dom} f-C)$. In other words, the regularity condition (23) does not imply (22).

Let $X, Y$ be finite dimensional spaces. Note that the convex programming problem considered in Section 4 is a particular case of problem (21) with $X=X \times Y$ and $C=\operatorname{Gr} F$. From Proposition 5.1 we obtain the following statement.

Proposition 5.2. Let $X, Y$ be finite dimensional spaces. The regularity conditions (i) and (ii) of Corollary 4.1 are weaker than (20).

Remark 5.1. In the case $X, Y$ are Banach spaces. It is still unclear to us whenever the regularity conditions (i) and (ii) of Corollary 4.1 are weaker than (20), or not?

The assertions of Theorem 4.1 and 4.2 are similar to those in [16, Theorems 5.1]. By imposing the strong convexity requirement on (8), we obtain corresponding equalities.

\section{Conclusions}

We have obtained exact formulas for the subdifferential and singular subdifferential of the marginal function for parametric convex programs by using intersection formulas and 
appropriate assumptions. Comparions with some known results have been given.

Acknowledgements. The research of Duong Thi Viet An was supported by Thai Nguyen University of Sicences, Vietnam. The research of Abderrahim Jourani was partially supported by the EIPHI Graduate School (contract ANR-17-EURE-0002). The authors would like to thank the two anonymous referees for their very careful readings and valuable suggestions which have helped to greatly improve the presentation.

\section{References}

1. An, D.T.V., Yao, J.-C.: Further results on differential stability of convex optimization problems. J. Optim. Theory Appl. 170, 28-42 (2016)

2. An, D.T.V., Yen, N.D.: Differential stability of convex optimization problems under inclusion constraints. Appl. Anal. 94, 108-128 (2015)

3. Aubin, J.-P.: Optima and Equilibria, An Introduction to Nonlinear Analysis. Springer, New York (2010)

4. Barbara A., Jourani, A.: Error bound characterizations of Guignard's constraint qualification in convex programming, 2021, submitted, https://hal.archives-ouvertes.fr/hal$03225245 /$

5. Bonnans, J.F., Shapiro, A.: Perturbation Analysis of Optimization Problems. Springer, New York (2000)

6. Borwein, J., Lucet, Y., Mordukhovich, B.: Compactly epi-Lipschitzian convex sets and functions in normed spaces. J. Convex Anal. 2, 375-393 (2000)

7. Borwein, J.M., Strojwas, H.M.: Tangential approximations. Nonlinear Anal. 12, 13471366 (1985)

8. Burke, J.V., Ferris, M.C., Qian, M.: On the Clarke subdifferential of the distance function of a closed set. J. Math. Anal. Appl. 166, 199-213 (1992) 
9. Clarke, F.H.: A new approach to Lagrange multipliers. Math. Oper. Res. 1, 165-174 (1976)

10. Clarke, F.H.: Optimization and Nonsmooth Analysis. Wiley, New York (1983)

11. Gauvin, J., Dubeau, F.: Differential properties of the marginal function in mathematical programming. Math. Program. Study 19, 101-119 (1982)

12. Gollan, B.: On the marginal function in nonlinear programming. Math. Oper. Res. 9, 208-221 (1984)

13. Henrion, R., Jourani, A.: Subdifferential conditions for calmness of convex constraints. SIAM J. Optim. 13, 520-534 (2002)

14. Ioffe, A.D.: Approximate subdifferentials and applications III: The metric theory. Mathematika 36, 1-38 (1989)

15. Ioffe, A.D., Tihomirov, V.M.: Theory of Extremal Problems. North-Holland Publishing Company, Amsterdam (1979)

16. Jourani, A.: Intersection formulae and the marginal function in Banach spaces. J. Math. Anal. Appl. 192, 867-891 (1995)

17. Jourani, A.: On a class of compactly epi-Lipschitzian sets. Nonlinear Anal. 54, 471-483 (2003)

18. Mordukhovich, B.S.: Variational Analysis and Generalized Differentiation. Volume I: Basic Theory, Volume II: Applications. Springer, Berlin (2006)

19. Mordukhovich, B.S., Nam, N.M., Yen, N.D.: Subgradients of marginal functions in parametric mathematical programming. Math. Program. Ser. B, 116, 369-396 (2009)

20. Thibault, L.: On subdifferentials of optimal value functions. SIAM J. Control Optim. 29, 1019-1036 (1991) 
21. Zănlinescu, C.: Convex Analysis in General Vector Spaces. World Scientific, New Jersey-London-Singapore-Hong Kong (2002) 